\title{
Is autonomic function associated with left ventricular systolic function in Chagas heart disease patients undergoing treatment for heart failure?
}

\author{
Lotte Raadschilders ${ }^{[1],[2]}$, Manoel Otavio da Costa Rocha ${ }^{[1]}$, Lidiane Sousa ${ }^{[1]}$, \\ Jan Nouwen ${ }^{[2]}$ and Antonio Luiz Ribeiro[1]
}

[1]. Faculdade de Medicina e Hospital das Clínicas, Universidade Federal de Minas Gerais, Belo Horizonte, MG. [2]. Department of Medical Microbiology en Infectious Diseases, Erasmus MC, Rotterdam, The Netherlands.

\begin{abstract}
Introduction: The association between cardiac autonomic and left ventricular (LV) dysfunction in Chagas disease (ChD) is controversial. Methods: A standardized protocol that includes the Valsalva maneuver, a respiratory sinus arrhythmia (RSA) test, and an echocardiographic examination was used. Spearman correlation coefficients (rho) were used to investigate associations. Results: The study population consisted of $118 \mathrm{ChD}$ patients undergoing current medical treatment, with an average LV ejection fraction of $51.4 \pm 2.6 \%$. The LV ejection fraction and diastolic dimension were correlated with the Valsalva index (rho $=0.358$, $\mathrm{p}<0.001$ and $\mathrm{rho}=-0.266, \mathrm{p}=0.004$, respectively) and the $\mathrm{RSA}(\mathrm{rho}=0.391, \mathrm{p}<0.001$ and rho $=-0.311, \mathrm{p}<0.001$, respectively). Conclusions: The impairment of LV function is directly associated with a reduction of cardiac autonomic modulation in $\mathrm{ChD}$.
\end{abstract}

Keywords: Chagas disease. Autonomic function. Valsalva index.

Chagas disease $(\mathrm{ChD})$ continues to be a major health problem in Latin America despite the implementation of prevention programs and a reduction in its prevalence ${ }^{1}$. Dysautonomia is a well-known complication of $\mathrm{ChD}$, which may occur early during the natural history of the disease ${ }^{2}$. The clinical significance of cardiac autonomic dysfunction in $\mathrm{ChD}$ is not completely understood, but it has been related to the risk of sudden death ${ }^{3}$.

In other cardiac diseases, the degree of vagal dysfunction is directly related to the decrease of the left ventricular ejection fraction (LVEF) ${ }^{4}$. In ChD, parasympathetic dysfunction precedes left ventricular (LV) dysfunction ${ }^{2}$, and autonomic and myocardial dysfunction may be independent phenomena. Previous studies have yielded controversial results regarding the relationship between vagal dysfunction and LV function ${ }^{5-9}$.

We hypothesize that autonomic function is associated with left ventricular function in $\mathrm{ChD}$. In other words, although patients with normal LV function may have autonomic impairment, those with more severe LV function impairment may have a more pronounced reduction of autonomic control of the heart. Therefore, we evaluated the correlation between autonomic indexes (the respiratory sinus arrhythmia [RSA] test and Valsalva maneuver) and LV function measurements, namely, $\mathrm{LVEF}$ and $\mathrm{LV}$ diastolic diameter $(\mathrm{LVd})$ values.

\footnotetext{
Address to: Dr. Antonio Luiz Ribeiro. FM/UFMG. Rua Campanha 98/101, Carmo, 30310-770 Belo Horizonte, MG, Brasil.

Phone: 5531 3409-9379

e-mail: tom@hc.ufmg.br

Received 11 February 2013

Accepted 12 August 2013
}

In this study, we combined the data from two different previous studies that used the same clinical protocol ${ }^{8,10}$ to increase the statistical power of analysis and to evaluate the patients representing the entire spectrum of LV function. The data were collected from patients who were referred from blood banks or primary care units to the Outpatient Reference Center at the University Hospital of the Universidade Federal de Minas Gerais (UFMG). The data used in this study were previously used in other studies related to Chagas disease and autonomic function, and the protocols were approved by the Ethics Committee of UFMG during the period 1997-2004,10. At the time of recruitment, the patients provided informed consent for data collection and use. After a confirmed diagnosis of $\mathrm{ChD}$ using at least two different serologic tests, the patients were evaluated using a standardized protocol that included a questionnaire (including a list of current medication), the Valsalva maneuver, an RSA test, and an echocardiographic study.

All of the autonomic tests were performed in a quiet room. Patients were still taking their current medications on a normal basis but fasted for $2 \mathrm{~h}$ and abstained from caffeine use, smoking, and medication for at least $12 \mathrm{~h}$ before the tests. Before the Valsalva maneuver was performed, all of the patients were provided instructions on how to perform it and were allowed to practice it a few times. The patients were instructed to blow into a mouthpiece with a closed glottis following deep inspiration. The mouthpiece was connected to a tube that was connected to an aneroid manometer to maintain an intraoral pressure of $40 \mathrm{mmHg}$ for $15 \mathrm{sec}$. The Valsalva maneuver was considered to be effective when facial plethora, neck vein distension, and abdominal muscle contraction were observed. An electrocardiogram was performed, and the electrocardiogram recording was analyzed in a blinded fashion. The Valsalva ratio was 
calculated as the ratio of the longest $\mathrm{R}-\mathrm{R}$ interval recorded during phase 4 to the shortest R-R interval recorded during phase $2^{11}$.

For the RSA test, the subjects were instructed to breathe deeply at a rate of 6 cycles per minute with the help of a metronome, and the R-R interval was recorded with an electrocardiogram. The E/I ratio (the ratio of the longest expiratory R-R interval to the smallest inspiratory interval) was calculated for each cycle, and the mean value was calculated ${ }^{2}$.

The echocardiographic study was performed by experienced echocardiographers, using a standardized protocol as previously described ${ }^{8,10}$. For this study, we used the data associated with LV function, specifically LVEF and LVd.

Data were analyzed using Statistical Package for the Social Sciences (SPSS) software, version 18 (SPSS Inc. Released 2009, Chicago, IL, USA), at the University Hospital of UFMG in Belo Horizonte. Continuous variables are described as means and standard deviations, and categorical variables are described as percentages. We analyzed the association between the autonomic function indexes and left ventricular function measurements using Spearman correlation coefficients (rho) because of the asymmetric distribution of most of the variables.

The total population in this study consisted of $118 \mathrm{ChD}$ patients, with complete data derived from 2 different databases. Our population, with a mean age of $49.9( \pm 8.5)$ years, consisted of $58 \%$ males, with an average LVEF of $51.4 \%( \pm 14.8)$. The majority of our population belonged to Goldman functional class I, and most used at least one medication for the treatment of heart failure, such as angiotensin-converting enzyme inhibitors or amiodarone (Table 1).

Figure 1 displays the association between the LV measurements and autonomic indexes. The LVEF is correlated with the Valsalva index ( $\mathrm{rho}=0.358, \mathrm{p}<0.001)$ and the RSA $(\mathrm{rho}=0.391, \mathrm{p}<0.001)$. The strength of the correlation between autonomic indexes and LVd is similar to that between the autonomic indexes and LVEF; however, the correlation between autonomic indexes and LVd is opposite to that between the autonomic indexes and LVEF (Valsalva index $[\mathrm{rho}=-0.266, \mathrm{p}=0.004]$ and RSA [rho $=-0.311$, $\mathrm{p}=0.001]$ ). Table 2 shows the Spearman correlation coefficients for the association between LV measurements and autonomic indexes stratified by the sample of origin (datasets 1 and 2). Although some variation of the magnitude of the association could be observed, no systematic bias could be detected.

Our data indicated that the autonomic function was directly correlated with left ventricular function in $\mathrm{ChD}$. Previous studies on this subject have produced contradictory results. Ribeiro et al. ${ }^{6}$ and Villar et al. ${ }^{7}$ reported that the intensity of vagal dysfunction is not related to the presence of cardiomyopathy, whereas Davila et al. ${ }^{5}$, Tundo et al. ${ }^{8}$, and Vasconcelos and Junqueira Junior ${ }^{9}$ reported an association between autonomic indexes and LV function. The reasons for the absence of a significant correlation in some of the previous studies are most likely related to a small sample size and lack of patients with severe LV dysfunction. In the present study, a wider range of LV function values provided a better understanding of the effects of $\mathrm{ChD}$ on $\mathrm{EF}$. Our results are in accordance with the findings of studies in patients with other heart diseases, in which a direct relationship between LV function and autonomic heart control
TABLE 1 - Clinical and functional features of Chagas disease patients.

\begin{tabular}{|c|c|c|}
\hline Variables & & \\
\hline Age (years), mean (SD) & 49.9 & $(8.5)$ \\
\hline Male $(\%)$ & 0.58 & \\
\hline \multicolumn{3}{|l|}{ Goldman functional class ( $\%)$} \\
\hline I & 74.2 & \\
\hline II & 18.9 & \\
\hline III & 6.8 & \\
\hline Amiodarone (\%) & 33.0 & \\
\hline Beta blockers (\%) & 14.0 & \\
\hline Digoxin $(\%)$ & 9.0 & \\
\hline Angiotensin-converting enzyme inhibitors (\%) & 62.0 & \\
\hline Diuretics (\%) & 49.0 & \\
\hline LVEF (\%), mean (SD) & 51.4 & $(14.8)$ \\
\hline LVEF greater than $45 \%(\%)$ & 65.0 & \\
\hline Left ventricular diastolic diameter (mm), mean (SD) & 57 & $(8.0)$ \\
\hline Valsalva index, mean (SD) & 1.41 & $(0.24)$ \\
\hline Respiratory sinus arrhythmia, mean (SD) & 1.13 & $(0.10)$ \\
\hline
\end{tabular}

SD: standard deviation; LVEF: left ventricular ejection fraction.

has been observed ${ }^{4}$. Nevertheless, in contrast to the studies of other cardiopathies, previous studies in our group indicate that in $\mathrm{ChD}$, autonomic dysfunction precedes $\mathrm{LV}$ dysfunction, and this phenomenon may be observed even in asymptomatic patients with cardiopathy ${ }^{2}$. Indeed, specific processes may occur in $\mathrm{ChD}$ leading to parasympathetic impairment, which occurs because of the destruction of the vagal ganglia and nerve terminations due to the inflammatory process ${ }^{12}$ and the inhibition of muscarinic receptors by autoantibodies ${ }^{13}$. However, our data indicate that as LV dysfunction and heart failure progress, vagal heart dysfunction worsens because of mechanisms that appear to be common with those of other heart diseases.

The strength of the present study is the use of a large database with a full spectrum of LV ejection fractions and the standardization of the autonomic tests. A limitation of the present study is the use of two different databases and the fact that all of the patients are from the same hospital; thus, possible regional differences in the $T$. cruzi strains were not considered. The sample included a few elderly patients and many subjects taking cardiovascular medications, which can potentially modify autonomic indexes. Moreover, it should be stressed that despite the fact that there was a significant and linear correlation between autonomic function and left ventricular function in $\mathrm{ChD}$, the correlation coefficients were relatively low, indicating that this association is relatively weak and that LV impairment may be associated but not necessarily be the cause of vagal dysfunction. 

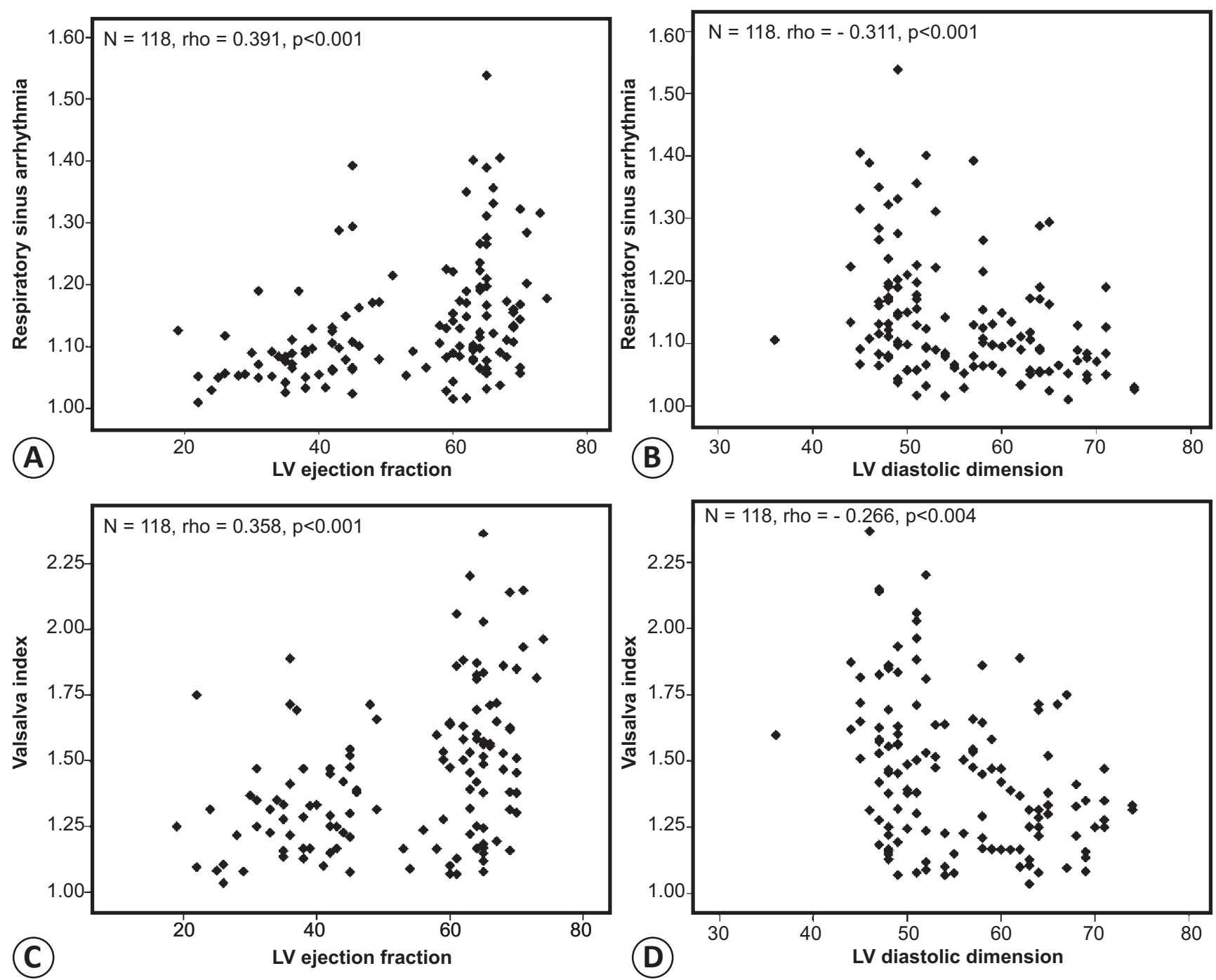

FIGURE 1 - Association between left ventricular measurements and autonomic tests: (a) left ventricular (LV) ejection fraction and respiratory sinus arrhythmia, (b) LV ejection fraction and Valsalva index, (c) LV diastolic diameter and respiratory sinus arrhythmia, and (d) LV diastolic diameter and Valsalva index.

TABLE 2 - Association between left ventricular measurements (left ventricular ejection fraction and diastolic diameter) and autonomic tests (respiratory sinus arrhythmia and Valsalva index) in the two datasets of Chagas heart disease patients used in this study.

\begin{tabular}{|c|c|c|c|c|c|}
\hline & & \multicolumn{2}{|c|}{ Respiratory sinus arrhythmia } & \multicolumn{2}{|c|}{ Valsalva index } \\
\hline & & rho & p-value & rho & p-value \\
\hline \multirow[t]{2}{*}{ Left ventricular ejection fraction } & Dataset 1 & 0.247 & 0.029 & 0.355 & 0.002 \\
\hline & Dataset 2 & 0.41 & 0.01 & 0.216 & 0.18 \\
\hline Left ventricular diastolic diameter & Dataset 1 & -0.182 & 0.11 & -0.261 & 0.024 \\
\hline
\end{tabular}

Rho: Spearman correlation coefficients. 
In conclusion, autonomic function is related to left ventricular function in $\mathrm{ChD}$, although patients with normal $\mathrm{LV}$ function could have vagal impairment. The prognostic significance of autonomic dysfunction is still unknown and warrants further investigation in prospective studies.

\section{ACKNOWLEDGMENTS}

We acknowledge F. Tundo for collecting the data that we used to analyze these correlations.

\section{CONFLICT OF INTEREST}

The authors declare that there is no conflict of interest.

\section{FINANCIAL SUPPORT}

Lotte Raadschilders is supported by the Dutch Heart Foundation. Manoel Otavio da Costa Rocha and Antonio Luiz Ribeiro have scholarships from Conselho Nacional de Desenvolvimento Cientifico e Tecnológico (CNPq), and from Fundação de Amparo à Pesquisa de Minas Gerais (FAPEMIG) and Lidiane Sousa (LS) has a scholarship from Financiadora de Estudos e Projetos (FINEP).

\section{REFERENCES}

1. Coura JR, Borges-Pereira J. Chagas disease: What is known and what should be improved: a systemic review. Rev Soc Bras Med Trop 2012; 45:286-296.
2. Ribeiro AL, Moraes RS, Ribeiro JP, Ferlin EL, Torres RM, Oliveira E, et al. Parasympathetic dysautonomia precedes left ventricular systolic dysfunction in Chagas disease. Am Heart J 2001; 141:260-265.

3. Junqueira-Junior LF. Insights into the clinical and functional significance of cardiac autonomic dysfunction in Chagas disease. Rev Soc Bras Med Trop 2012; 45:243-252.

4. Mortara A, La Rovere MT, Pinna GD, Prpa A, Maestri R, Febo O, et al. Arterial baroreflex modulation of heart rate in chronic heart failure: clinical and hemodynamic correlates and prognostic implications. Circulation 1997; 96:3450-3458.

5. Davila DF, Donis JH, Navas M, Fuenmayor AJ, Torres A, Gottberg C. Response of heart rate to atropine and left ventricular function in Chagas' heart disease. Int J Cardiol 1988; 21:143-156.

6. Ribeiro AL, Lombardi F, Sousa MR, Lins Barros MV, Porta A, Costa Val Barros V, et al. Power-law behavior of heart rate variability in Chagas' disease. Am J Cardiol 2002; 89:414-418.

7. Villar JC, León H, Morillo CA. Cardiovascular autonomic function testing in asymptomatic $\mathrm{T}$. cruzi carriers: a sensitive method to identify subclinical Chagas' disease. Int J Cardiol 2004; 93:189-195.

8. Tundo F, Lombardi F, Rocha MC, Botoni F, Schmidt G, Barros VC, et al. Heart rate turbulence and left ventricular ejection fraction in Chagas disease. Europace 2005; 7:197-203.

9. Vasconcelos DF, Junqueira Júnior LF. Cardiac autonomic and ventricular mechanical functions in asymptomatic chronic chagasic cardiomyopathy. Arq Bras Cardiol 2012; 98:111-119.

10. Sousa L, Rocha MO, Britto RR, Lombardi F, Ribeiro AL. Chagas disease alters the relationship between heart rate variability and daily physical activity. Int J Cardiol 2009; 135:257-259.

11. Oliveira E, Ribeiro AL, Assis Silva F, Torres RM, Rocha MO. The Valsalva maneuver in Chagas disease patients without cardiopathy. Int $\mathrm{J}$ Cardiol 2002; 82:49-54.

12. Junqueira Júnior LF, Beraldo PS, Chapadeiro E, Jesus PC. Cardiac autonomic dysfunction and neuroganglionitis in a rat model of chronic Chagas' disease. Cardiovasc Res 1992; 26:324-329.

13. Ribeiro AL, Giménez LE, Hernández CC, Carvalho AC, Teixeira MM, Guedes VC, et al. Early occurrence of anti-muscarinic autoantibodies and abnormal vagal modulation in Chagas disease. Int J Cardiol 2007; 117:59-63. 